\title{
Experimental Observation of the Amplitude Death Effect in Two Coupled Nonlinear Oscillators
}

\author{
R. Herrero, M. Figueras, J. Rius, F. Pi, and G. Orriols \\ Departament de Física, Universitat Autònoma de Barcelona, 08193 Bellaterra, Spain
} (Received 22 October 1999)

\begin{abstract}
The amplitude death phenomenon has been experimentally observed with a pair of thermo-optical oscillators linearly coupled by heat transfer. A parametric analysis has been done and compared with numerical simulations of a time delayed model. The role of the coupling strength is also discussed from experimental and numerical results.
\end{abstract}

PACS numbers: 05.45.Xt, 42.50.Ar

Systems of interacting nonlinear units have been of much interest because of their abundance in nature $[1,2]$. The coupling introduces new possible behaviors to the whole system, especially for oscillating units. Synchronization in chaotic lasers [3], in neurons [2] and between the heartbeat and ventilation [4], or phase locking in insect chirping [1] are some of the observed behaviors that can be described by phase models considering a weak coupling. On the other hand, effects associated with the amplitude of the different units are expected for strong couplings. One of the most intriguing effects is the so-called amplitude death, dealing with the absence of oscillations for the coupled system while each subsystem oscillates when isolated. Two kinds of amplitude death have been considered. In one of the types the coupling creates a saddle-node pair of fixed points on the limit cycle of the entrained oscillations [5], while the second one is associated with a more common bifurcation - the Hopf bifurcation - in which the oscillation amplitudes are damped out up to reach a steady state [6]. The relevance of the second kind of amplitude death and the important role assumed by the time delay in the coupling of oscillators have been recently noted due to its possible application in physics, chemistry, medicine, and biology [7]. The effect has been predicted to occur for two or more identical or different coupled oscillators [6,8-10], but it has not been experimentally observed until now [7].

Here, we present the experimental observation of the amplitude death phenomenon with two optothermal oscillators, located on the same interferometric device and thermally coupled. The relation with the coupling effects on the Hopf bifurcation of the whole system is clearly stated by means of a parametric analysis as a function of the coupling strength and the relative light irradiation of the coupled units. The experimental results are compared with simulations of a time delayed model.

The nonlinear system is based on the so-called optothermal bistability with localized absorption (BOITAL) and consists of a Fabry-Pérot cavity where the input mirror is a partially absorbing film, the rear mirror is a high reflection dielectric coating, and the spacer is made of two transparent layers with opposite thermo-optic effects [11]. Such a kind of system presents a multiple steady-state solution associated with the periodic interferometric function and can experience a supercritical Hopf bifurcation due to the competition and time delay between the contributions of the two layers to the light phase shift [11]. With this configuration and increasing the input power, the system destabilizes at the first stable branch yielding a limit cycle, which grows by approaching the neighboring saddle point and a homoclinic connection may occur. After the connection the limit cycle is destroyed and the flow escapes towards an upper node branch where a similar behavior takes place.

For a transversally extended optical device, different oscillators separated by a distance $d$ can be created by using parallel light beams. The nonlinear elements are coupled by tranverse heat diffusion and the separation distance may be used to adjust the coupling degree. The oscillators have the same cavity parameters, although slight differences in the optical path may occur due to cavity misalignments, and significant differences may be introduced with the incident light powers. This work describes results for two coupled oscillators only.

Experiments were performed with a device made of $400 \mu \mathrm{m}$ of glass and $100 \mu \mathrm{m}$ of sunflower oil with effective thermo-optical effects of $10^{-5} \mathrm{~K}^{-1}$ and $-3.4 \times$ $10^{-4} \mathrm{~K}^{-1}$, respectively. The cavity mirrors were a $7 \mathrm{~nm}$ nickel-chrome film and a high reflection dielectric multilayer. The device was irradiated with a continuous-wave $\mathrm{Ar}^{+}$laser beam split in two, and each beam was focalized to a $50 \mu \mathrm{m}$ diameter spot on the device. The incident powers are denoted by $P_{e}^{a}$ and $P_{e}^{b}$ and the ratio $\gamma=P_{e}^{a} / P_{e}$, with $P_{e}=P_{e}^{a}+P_{e}^{b}$, is used to characterize the relative illumination of the oscillators.

The amplitude death was observed for short separating distances and the upper part of Fig. 1 presents time evolutions illustrating this effect for $d=200 \mu \mathrm{m}$. Both oscillators were illuminated with similar powers above the individual Hopf bifurcations but, nevertheless, the system remained in a stable rest state. Blocking any one of the input beams, oscillations appeared in the reflected power of the illuminated unit while they disappeared when both beams were replaced again. The transient to the stable steady state was through antiphase damped oscillations slightly faster than those of the uncoupled units. The lower part of Fig. 1 presents a behavior that could be called amplitude semideath, observed for lower and more 


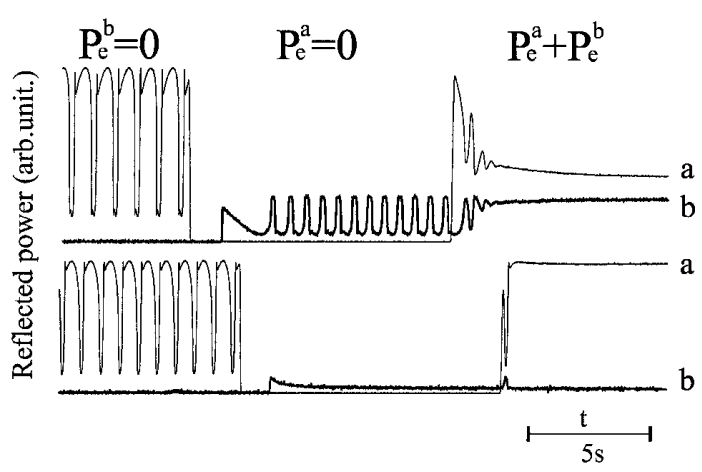

FIG. 1. Response of two uncoupled and coupled BOITAL oscillators for $d=200 \mu \mathrm{m}$. The upper time evolutions, for $\gamma=$ 0.49 and $P_{e}=62.1 \mathrm{~mW}$, show the amplitude death effect and the lower ones, for $\gamma=0.73$ and $P_{e}=38.3 \mathrm{~mW}$, show the semideath effect.

asymmetric input powers. In this case, the system response was a stable steady state when the highest power input beam was blocked and a limit cycle when the lowest power input beam was blocked. The oscillations disappeared when both beams were replaced.

The diagram of Fig. 2 shows the local and global bifurcations of the coupled system observed when the total input power $P_{e}$ and the ratio $\gamma$ were scanned. The extreme values $\gamma=0$ and $\gamma=1$ characterize the isolated subsystems $b$ and $a$, respectively, by showing their corresponding $P_{e}$ oscillation intervals delimited by the Hopf and homoclinic bifurcations. The similar individual oscillation thresholds denote a small difference between the cavity optical paths of the two units. Thus, the bifurcation diagram of Fig. 2 describes two nearly similar BOITAL cavities as a function of their relative illumination. The crossed hyperbolic curves project the individual Hopf bi-

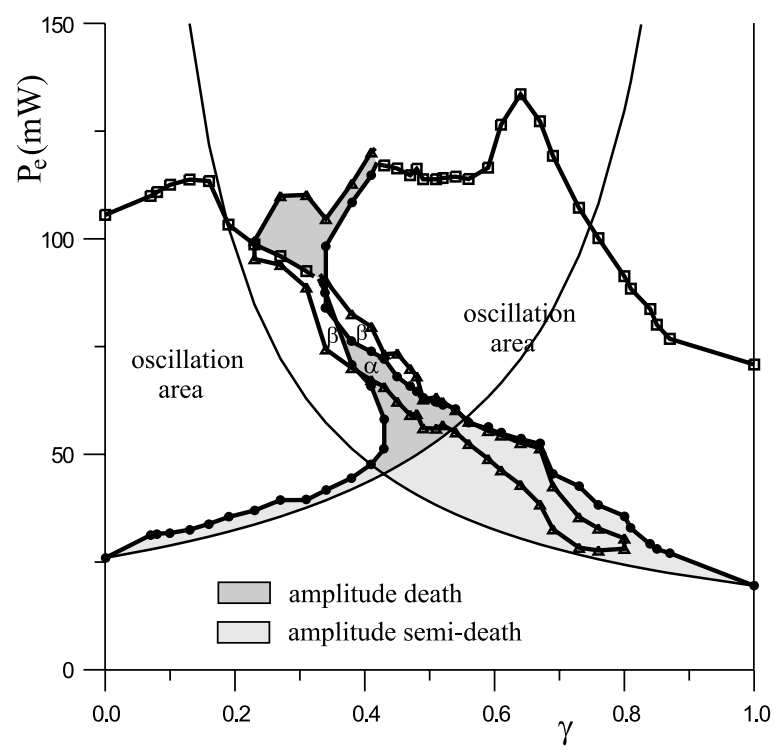

FIG. 2. Bifurcation diagram in the $\gamma-P_{e}$ parameter plane for the oscillators of Fig. 1. indicates Hopf bifurcations, $\triangle$ saddle-node bifurcations, and $\square$ homoclinic bifurcations. furcations over the whole diagram by taking into account the $\gamma$ factor and permit the direct comparison between the coupled and uncoupled systems. The oscillation area of the coupled system appears divided in two balloons, each one defined vertically by either two supercritical Hopf bifurcations or a Hopf and a homoclinic bifurcation. The balloons appear slightly superposed but in other cases they may be clearly separated.

The amplitude death effect occurs in the nonoscillating zones above both hyperbolic curves, and the semideath effect in the nonoscillating zones above one hyperbolic curve only. The simplest situation of amplitude death happens near the crossing of hyperbolic curves, where each subsystem alone would oscillate near its Hopf bifurcation. This is the typical situation considered in the theoretical works [8-10], and the upper signals of Fig. 1 correspond to it. The death phenomenon occurs because the coupling displaces the Hopf bifurcation of the whole system with respect to those of the individual oscillators. Notice, however, that in the case of Fig. 2 the death regions involve also a tongue-shaped curve of saddle-node (S-N) bifurcations, which contains two codimension-two cusp points at $\left(\gamma, P_{e}\right)$ equal to $(0.22,95 \mathrm{~mW})$ and $(0.8,30 \mathrm{~mW})$. This curve defines a bistable zone where the two steady states existing at the two sides coexist together. In the death region denoted by $\alpha$ both steady states are stable, while in the $\beta$ regions one of them has become unstable by yielding an oscillatory state. Thus, in the $\beta$ regions the whole system may be either oscillating or dead depending on the previous history, i.e., the one or the other state will be obtained by blocking and replacing again the illumination of either one or another subsystem. In the semideath region the tongue contains two stable steady states. The enrichment of the steady-state solution is a characteristic feature of the coupled systems [6] and the coexistence of states in the death phenomena has been already detected in models of coupled oscillators $[6,8]$.

The BOITAL cavity is well described by the homogeneous heat equation subject to a nonlocal and nonlinear boundary condition [11], and it can be reduced to a dimensionless model of order equal to the number of layers [12]. In this model the variables, $\psi_{j}$, are the light phase shifts due to temperature rises in each layer. The $\psi_{j}$ are proportional to the averaged temperature and to the thermo-optic coefficient, $\eta_{j}$, of the corresponding layers. According to this model we describe the pair of thermally coupled two-layer oscillators as follows:

$$
\begin{aligned}
\frac{d \psi_{1}^{a}}{d t}= & -b_{11} \psi_{1}^{a}-b_{12} \psi_{2}^{a}+G_{1} A\left(\psi^{a}\right) \mu_{e}^{a} \\
& +c_{1} \psi_{1}^{b}\left(t-\tau_{1}\right)-\left(c_{1}+c_{2}\right) \psi_{1}^{a} \\
\frac{d \psi_{2}^{a}}{d t}= & -b_{21} \psi_{1}^{a}-b_{22} \psi_{2}^{a}+G_{2} A\left(\psi^{a}\right) \mu_{e}^{a} \\
& +c_{2} \frac{\eta_{2}}{\eta_{1}} \psi_{1}^{b}\left(t-\tau_{2}\right)
\end{aligned}
$$


and the same equations with superindexes $a$ and $b$ exchanged. $\psi^{x}=\psi_{0}^{x}+\sum_{j=1}^{2} \psi_{j}^{x}$ is the total round-trip phase shift for oscillator $x, \psi_{0}^{x}$ is the phase shift in the absence of laser heating, and $\mu_{e}^{x}$ is the normalized incident light power. The nonlinear function $A(\psi)$ describes the light interference within the absorbing mirror and depends on the mirror parameters only. The rates $b_{j i}$ describe the longitudinal thermal coupling between layers and the associated diffusion times. $G_{j}$ characterizes the effective contribution of the $j$ th layer to the phase shift variations. $A(\psi), b_{j i}$, and $G_{j}$ are common to both oscillators and their expressions as a function of the physical parameters are given in [11,12]. The transverse coupling between oscillators is phenomenologically considered by adding linear terms describing the heat flow from the layer next to the absorbing film towards the two layers of the neighbor. The coupling coefficients are $c_{1}=f \frac{K_{1}}{d^{2}}$ and $c_{2}=f \frac{K_{1}}{d^{2}+g_{1}^{2}}$, respectively. The finite speed of heat propagation is taken into account by introducing two time delays, $\tau_{1}=\frac{d^{2}}{D_{1}}$ and $\tau_{2}=\frac{d^{2}+g_{1}^{2}}{D_{1}}$, into the terms describing the heat arrival at the first and second layers, respectively. $D_{1}, K_{1}$, and $g_{1}$ are the thermal diffusivity, thermal conductivity, and thickness of the first layer, respectively, and $f$ is a constant factor relating heat transfer with phase variations. Notice, however, that a constant time delay does not account for the strong dispersion of thermal diffusion at low frequencies and therefore provides a limited description of the propagation process.

The numerical bifurcation diagram of Fig. 3 corresponds to the same parameter plane as in Fig. 2 and the other parameters close to the experimental ones. The bifurcation structure defining the oscillation areas and the death and semideath regions is qualitatively similar to the experimental case. The main difference concerns the bistable steady-state tongue, which appears smaller and with a reverse shape. The bifurcation diagram is very sensitive to the $\psi_{0}^{x}$ values and bistable tongues like that of Fig. 2 can be obtained, but we have not been able to reproduce both the steady-state and the oscillatory instabilities simultaneously. Figure 3 presents also time evolutions illustrating the amplitude death.

The amplitude death is expected only for strong couplings $[8,9]$. In Fig. 4, the distance $d$ between the two oscillators was scanned to change the coupling strength. Both oscillators were maintained under equal illumination $(\gamma=0.5)$ while the $P_{e}$ scannings were done. The light beam inclinations were adjusted for each distance to achieve equal individual responses. The power of the Hopf bifurcation of the isolated oscillators $\left(P_{H}^{a}\right.$ and $\left.P_{H}^{b}\right)$ had almost the same value for all distances and the oscillation frequency remained at about $2.25 \mathrm{~Hz}$ within $1.5 \%$. Thus, the diagram characterizes a coupled pair of very similar oscillators (including the incident power) as a function of $d$, which modifies both the coupling strength and time delays in an opposite sense. Notice first the S-N bifur-

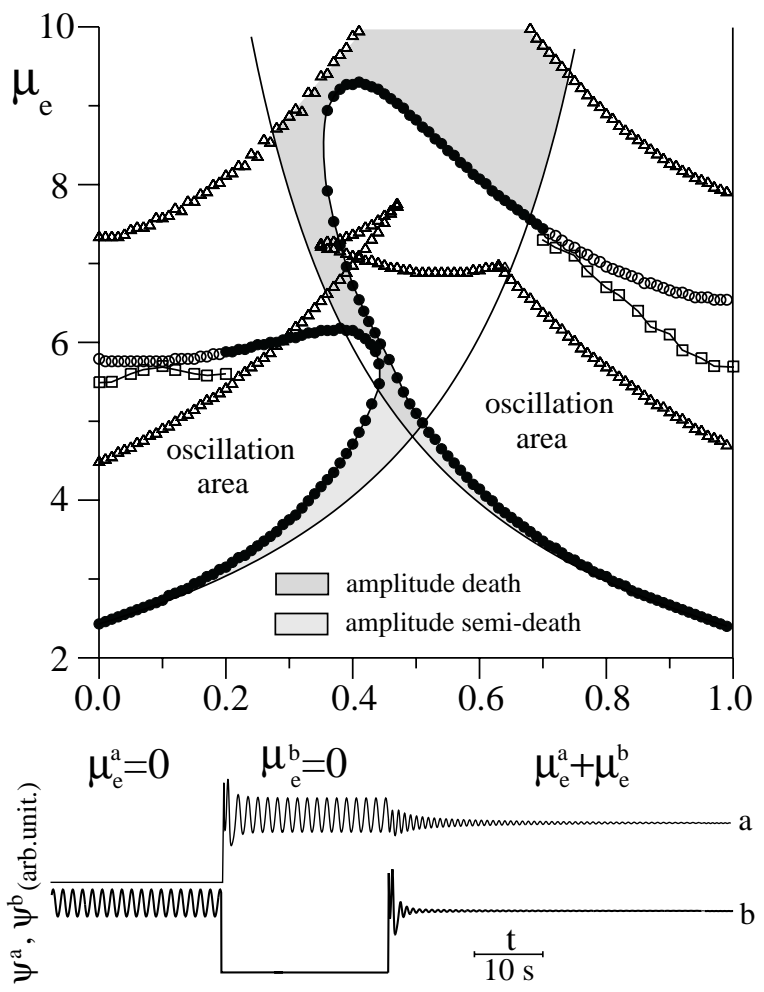

FIG. 3. Numerical simulation with adimensional parameters: $\quad \eta_{1,2}=1,-10, \quad g_{1,2}=1,0.25, \quad K_{1,2}=D_{1,2}=1,0.1$, $h_{F}=h_{B}=1.0, \quad d=0.32, \quad f=0.2, \quad \psi_{0}^{a}=5.0, \quad \psi_{0}^{b}=5.1$. The symbols are the same as in Fig. 2. Time evolutions for $\gamma=0.5$ and $\psi_{e}=5.0$ in the amplitude death zone.

cation emerging from a codimension-two cusp point near $200 \mu \mathrm{m}$ and defining a bistable region. There are also two Hopf bifurcation curves denoted by $A$ and $B$, which occur on different stationary branches and define two oscillation areas. The amplitude death takes place over the horizontal level of the individual Hopf bifurcations and outside the oscillation areas of the coupled system. Notice the variety of regions with different coexisting states within the bistable tongue.

All the Hopf bifurcations of Fig. 4 yielding sustained oscillations with increasing the input power are supercritical, but the inverse Hopf bifurcation $B$ shows a subcritic character. The oscillation amplitude does not decrease during the transition but the average period increases towards a divergence (see lower part of Fig. 4) and small modulations appear between the oscillations. The steady state appears over the disappearing limit cycle and the lack of any jump excludes a homoclinic bifurcation. A similar transition observed in a chemical model was associated with a saddle-node bifurcation occurring just on the limit cycle [5]. In our case, the experimental results are not conclusive but this kind of bifurcation has not been found in the numerical simulations.

Extensive analysis of models based on the normal form for the supercritical Hopf bifurcation have shown the necessity of either oscillator dissimilarities or time delays in 


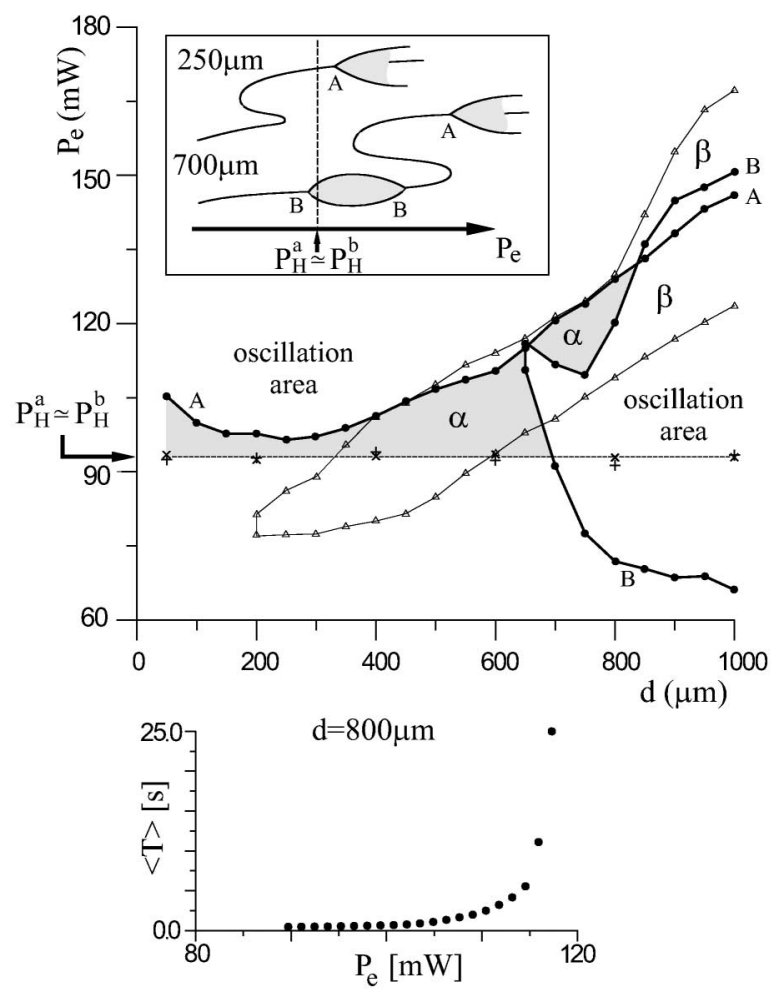

FIG. 4. Scan of the separating distance between oscillators for the same BOITAL device of Fig. 1 but with almost equal initial phase shift for the two subsystems and $\gamma=0.5$. Thick lines $A$ and $B$ correspond to Hopf bifurcations and thin lines denote saddle-node bifurcations. + and $\times$ denote Hopf bifurcations of the uncoupled units. The inset shows schemes of the observed bifurcations for two different distances. The lower part presents the period divergence in the transition to a death state occurring in the high part of the Hopf bifurcation $B$.

order to obtain the amplitude death effect [8-10]. Nevertheless, these analyses have been developed for a particular fixed point describing a homogeneous state of the two oscillators and whose behavior is perhaps not general enough. In fact, the amplitude death was first detected in a model of coupled chemical reactions without explicit time delays and for identical, nearly identical, and dissimilar oscillators [6]. In the identical case, the death was found in two inhomogeneous states arising from a pitchfork bifurcation of the homogeneous state. On the other hand, the BOITAL model for the same parameters as in Fig. 3, but with $\tau_{1}=\tau_{2}=0$ and $\psi_{0}^{a}=\psi_{0}^{b}(=5.1)$, produces amplitude death regions even larger than those of Fig. 3 and in this case the death state for $\gamma=0.5$ is homogeneous. The reason may be that the coupling terms in our model are not in the scalar form considered in the theoretical analysis [8]. In the experiment, the instantaneous coupling cannot be studied because the two light beams must remain separated to have distinct oscillators.
The introduction of time delayed terms is a simple way for considering the finite propagation speed, but a more accurate description can in principle be obtained by a proper reduction of the corresponding partial differential equation. For instance, in a BOITAL device both the coupling and intrinsic dynamics of each oscillator are due to heat diffusion and the reduction of the heat equation for the transversely uniform problem, solved in [12], might be generalized to the nonuniform case of the coupled system. Thus, for a given physical situation, the explicit presence of time delays into the equations is not obliged and the characteristic times of the problem can be contained in the structure and rate coefficients of the coupling terms.

In conclusion, the appearance of amplitude death states when two oscillating units are coupled has been experimentally verified. The parametric analysis has shown the relation of the death effect with the Hopf bifurcations of the uncoupled and coupled systems. The occurrence of this effect only for strong couplings and the possible bistability of death states has also been verified. The reported experiments are not conclusive about the role of the finite propagation speed of the coupling in the death phenomenon. In this respect it will be interesting to investigate the multiplicity of death islands predicted by the theory of the time delay induced death [13] in the parameter space defined by the coupling strength, time delay and oscillation frequency.

Financial support from the Spanish DGES under Grant No. PB98-0899 is acknowledged.

[1] E. Sismondo, Science 249, 55 (1990).

[2] R. C. Elson, A. I. Selverston, R. Huerta, N. F. Rulkov, M. I. Rabinovich, and H.D. I. Abarbanel, Phys. Rev. Lett. 81, 5692 (1998).

[3] R. Roy and S. Thornburg, Phys. Rev. Lett. 72, 2009 (1994).

[4] C. Schäfer, M. G. Rosenblum, J. Kurths, and H. Abel, Nature (London) 392, 239 (1998).

[5] M. Crowley and I. Epstein, J. Phys. Chem. 93, 2496 (1989).

[6] K. Bar-Eli, Physica (Amsterdam) 14D, 242 (1985).

[7] S. H. Strogatz, Nature (London) 394, 316 (1998).

[8] D. G. Aronson, G. B. Ermentrout, and N. Kopell, Physica (Amsterdam) 41D, 403 (1990).

[9] D. V. Ramana Reddy, A. Sen, and G. L. Johnston, Phys. Rev. Lett. 80, 5109 (1998).

[10] G. B. Ermentrout, Physica (Amsterdam) 41D, 219 (1990).

[11] J. I. Rosell, J. Farjas, R. Herrero, F. Pi, and G. Orriols, Physica (Amsterdam) 85D, 509 (1995).

[12] J. Farjas, J. I. Rosell, R. Herrero, R. Pons, F. Pi, G. Orriols, Physica (Amsterdam) 95D, 107 (1996).

[13] D. V. Ramana Reddy, A. Sen, and G. L. Johnston, Physica (Amsterdam) 129D, 15 (1999). 